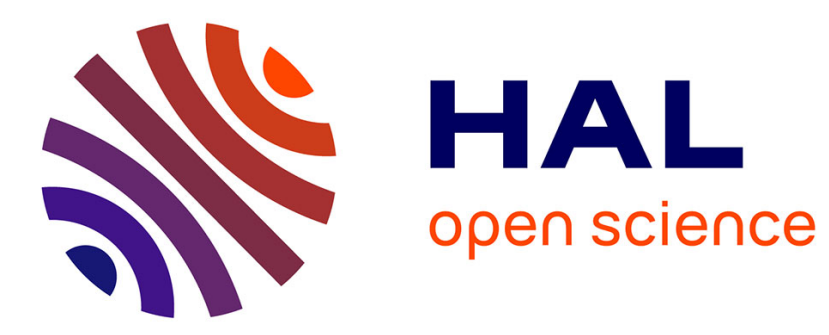

\title{
On the mechanical behaviour under cyclic loading of ceramic matrix composites
}

\author{
Alain Burr, François Hild, Frederick A Leckie
}

\section{To cite this version:}

Alain Burr, François Hild, Frederick A Leckie. On the mechanical behaviour under cyclic loading of ceramic matrix composites. Materials Science and Engineering: A, 1998, 250 (2), pp.256-263. 10.1016/S0921-5093(98)00599-1 . hal-02342757

\section{HAL Id: hal-02342757 \\ https://hal.science/hal-02342757}

Submitted on 4 Nov 2019

HAL is a multi-disciplinary open access archive for the deposit and dissemination of scientific research documents, whether they are published or not. The documents may come from teaching and research institutions in France or abroad, or from public or private research centers.
L'archive ouverte pluridisciplinaire HAL, est destinée au dépôt et à la diffusion de documents scientifiques de niveau recherche, publiés ou non, émanant des établissements d'enseignement et de recherche français ou étrangers, des laboratoires publics ou privés. 


\title{
On the mechanical behaviour under cyclic loading of ceramic matrix composites
}

\author{
Alain Burr ${ }^{a}$, François Hild ${ }^{a, *}$, Frederick A. Leckie ${ }^{b}$ \\ "Laboratoire de Mécanique et Technologie, ENS de Cachan/CNRS/Université Paris VI, 61 avenue du President Wilson, \\ F-94235 Cachan Cedex, France \\ ${ }^{b}$ Department of Mechanical and Environmental Engineering, College of Engineering, University of California, Santa Barbara, CA, USA
}

\begin{abstract}
In this paper, a constitutive law is presented to model the mechanical behaviour of ceramic matrix composites. It allows matrix-cracking, interfacial debonding, sliding and wear to be accounted for in the framework of continuum mechanics. Based upon micromechanical studies, a $1 \mathrm{D}$ and 2D model was derived. An application was performed on a $[0,90] \mathrm{SiC} / \mathrm{SiC}$ composite.
\end{abstract}

Keywords: Ceramic matrix composites; Cracking; Cyclic loading; Interfacial bedonding; Modeling

\section{Introduction}

Matrix-cracking and subsequent matrix-fibre interface debonding and sliding are the cause of a nonlinear stress-strain behaviour observed when loading ceramic matrix composites (CMCs). These basic mechanisms are the source of a reduction of the tangent Young's modulus, the presence of inelastic strain and hysteresis loop [1,2]. Furthermore, cycling under constant stress amplitude causes the total strain to increase with the number of cycles. This phenomenon is usually attributed to interfacial wear [3]. It is proposed to study these mechanisms in the framework of continuum mechanics and to derive constitutive laws suited for structural applications. In this connection, an attempt is made to base the formulation on the results of studies of the microstructural degradation mechanisms.

A continuum mechanics formulation applied to CMCs is written within the framework of the thermodynamics of irreversible processes [4,5]. The first step in establishing such a model is to identify the state variables which define the state of the material. The second step is to derive the expression of the state potential $\bar{\psi}$ in terms of the state variables and the third one is to determine the evolution laws of the internal variables. The state potential $\bar{\psi}$ is made up of the sum of two terms: viz. the elastic energy density $\bar{\psi}_{\mathrm{c}}$ and the stored energy density $\bar{\psi}_{\mathrm{s}}$. The elastic part is directly related to the applied load. The stored energy density is concerned with residual stress fields giving rise to macroscopic (or inelastic) strains with no applied load. The stored energy density is expressed as the total elastic energy density associated with the residual stress field. Therefore by considering two elastic steps, the total free energy density can be evaluated following a so-called 'cut and paste' technique introduced by Volterra [6]. This approach will be used to model the behaviour of unidirectional as well as $[0,90]$ layered fibre-reinforced CMCs.

In Section 2, the relevant microscopic quantities are introduced to analyse the above-described degradation mechanisms of CMCs. Based upon the results of Section 2, Section 3 deals with a macroscopic description of constitutive laws applicable to unidirectional CMCs. Section 4 is devoted to the extension of the results derived in Sections 2 and 3 to model the mechanical behaviour of $[0,90]$ layered and woven composites. In Section 5, the previous results are applied to analyse a $[0,90]$ fibre-reinforced $\mathrm{SiC} / \mathrm{SiC}$ composite. 


\section{Microscopic description of the degradation mechanisms}

The matrix cracks, which are assumed to be perpendicular to the fibre direction, cause a stiffness reduction when the stress is tensile. In the following, it was assumed that the whole matrix-cracking process occurs at load levels lower than the fibre breakage mechanism, and therefore only the former mechanism will be analysed. By using the same framework, the analysis of fibre breakage and pull-out can be found in Refs. [7,8]. Let us consider an elementary cell of length $2 L$, equal to the average crack spacing, consisting of two different materials (m) and (f) as shown in Fig. 1 [9], where $E$ is the Young's modulus of the unbroken composite, $E_{\mathrm{m}}$ that of part (m), and $E_{\mathrm{r}}$ that of part (f). There is a matrix crack at the centre of the cell. The presence of a matrix crack implies a potential energy density change $\Delta \bar{\varphi}_{\mathfrak{e}}$ given by

$\Delta \bar{\varphi}_{\mathrm{e}}=\frac{\bar{\sigma}^{2}}{E} \omega_{\mathrm{c}}$

where $\omega_{\mathrm{c}}$ is dependent on the crack density (i.e. $(\bar{R}-$ $\left.R_{f}\right) / L$, see Fig. 1) as well as on the fact whether cracks interact with each other or not. This first basic mechanism does not allow the storage of energy and therefore only influences the reversible (or elastic) part of the free energy density $\bar{\psi}_{\mathrm{e}}$.

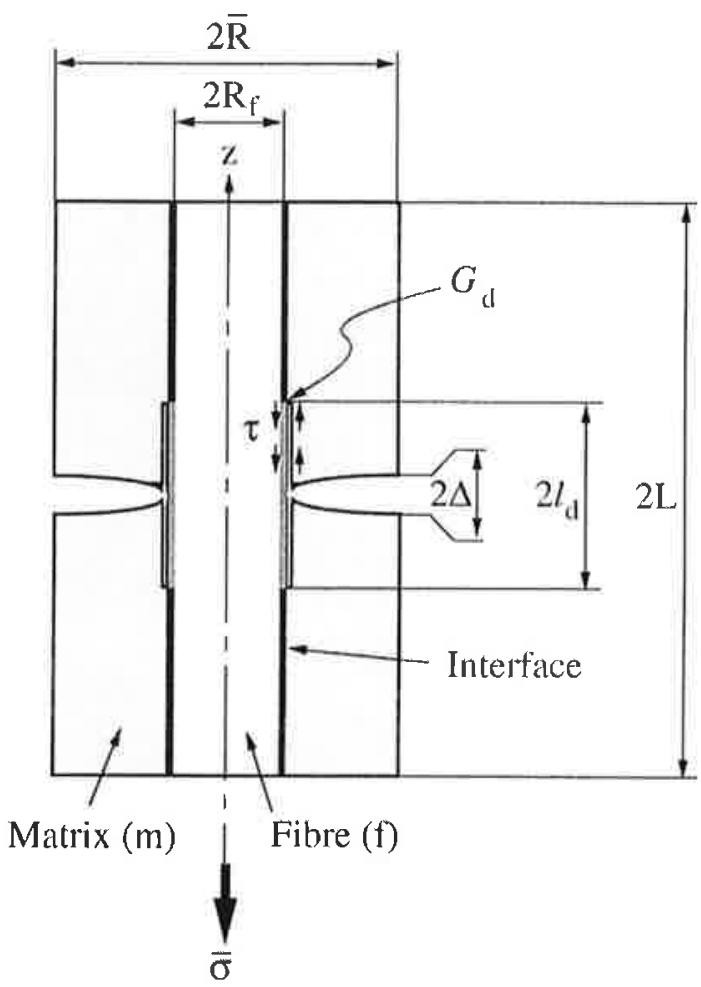

Fig. 1. Elementary cell with crack spacing $2 L$ and debond length $2 l_{\mathrm{d}}$.
Debonding followed by sliding gives rise to inelastic strains and hysteresis loops. To describe these phenomena, different models have been proposed [9-11]. They all consider a friction length $2 l_{\mathrm{f}}$ here assumed to be equal to the debond length $2 l_{\mathrm{d}}$ (Fig. 1). Similarly to cracking, which is a mode I mechanism, debonding per se cannot store energy. When debonding and slip occur simultaneously, a self-balanced microscopic stress field is involved. The corresponding strains in the matrix and in the fibre are denoted by $\Delta \epsilon_{\mathrm{m}}(z, L)$ and $\Delta \epsilon_{\mathrm{f}}(z, L)$, respectively, where $z$ is the current coordinate. These strain differences result from the 'cut and paste' procedure alluded to in Section 1. By application of the principle of virtual work [12], the overall inelastic strain $\bar{\epsilon}_{\text {in }}$ is expressed as

$\bar{\epsilon}_{\mathrm{in}}=\frac{1}{2 L} \int_{-L}^{L} \Delta \epsilon_{\mathrm{f}}(x, L) \mathrm{d} z$.

Since the additional stress field is self-balanced, the debonding and sliding processes are able to store energy. To fully characterise both mechanisms the knowledge of the corresponding stored energy density $\bar{\psi}_{\mathrm{s}}$ is crucial. It is expressed as the total elastic energy density associated with the residual stress field due to debonding and sliding

$\bar{\psi}_{\mathrm{s}}=\frac{1}{2 L} \int_{-L}^{L}\left\{f E_{\mathrm{f}} \Delta \epsilon_{\mathrm{f}}^{2}(z, L)+(1-f) E_{\mathrm{m}} \Delta \epsilon_{\mathrm{m}}^{2}(z, L)\right\} \mathrm{d} z$

where $f$ is the fibre volume fraction. It is worth noting that the expression of the stored energy is a priori only valid if the residual stress field is associated with debonding and sliding.

Under cyclic loading, wear may take place at the fibre-matrix interface because of sliding reversals [3]. An estimate of the effect of cyclic loading on the mechanical behaviour is possible when the influence of interfacial wear is included in the model. The effect of wear is a change of the residual stress field induced by debonding and sliding. Therefore as the number of cycles $N$ increases, there is a variation of the associated additional strains $\Delta \epsilon_{\mathrm{f}}(z, L, N)$ so that the inelastic strain becomes a function of the number of cycles $N$

$\bar{\epsilon}_{\text {in }}(N)=\frac{1}{2 L} \int_{-L}^{L} \Delta \epsilon_{\Gamma}(z, L, N) \mathrm{d} z$.

Similarly, there is a variation of the additional strains $\Delta \epsilon_{\mathrm{m}}(z, L, N)$ so that the corresponding stress fields are still self-balanced. Therefore the total stored energy density is also dependent on the number of cycles $N$ $\bar{\psi}_{\mathrm{s}}(N)$

$=\frac{1}{2 L} \int_{-L}^{L}\left\{f E_{\mathrm{f}} \Delta \epsilon_{\mathrm{f}}^{2}(z, L, N)+(1-f) E_{\mathrm{m}} \Delta \epsilon_{\mathrm{m}}^{2}(z, L, N)\right\} \mathrm{d} z$.

(5)

From the present analysis, three different quantities are obtained. A potential energy density change due to 
matrix-cracking $\Delta \bar{\varphi}_{e}$, inelastic strains $\bar{\epsilon}_{\mathrm{in}}(N)$ and a corresponding stored energy related to debonding, sliding and wear $\bar{\psi}_{\mathrm{s}}(N)$. These results are now used to model macroscopically the degradation mechanisms.

\section{Macroscopic description of the degradation mechanisms}

Matrix-cracking can be described by an internal damage variable $D$ to comply with the requirement of a fully dissipative mechanism (i.e. no energy can be stored). In a continuum mechanics framework, the presence of a crack results in a stiffness reduction defined by an internal damage variable $D[13,14]$. The Gibbs' elastic energy density of a damaged material is written as [15]

$\bar{\varphi}_{\mathrm{e}}(D)=\frac{\bar{\sigma}^{2}}{2 E(1-D)}$

so that the potential energy density change can be written as

$\Delta \bar{\varphi}_{\mathrm{e}}=\bar{\varphi}_{\mathrm{e}}(D)-\bar{\varphi}_{\mathrm{e}}(D=0)=\frac{\bar{\sigma}^{2}}{2 E} \frac{D}{1-D}$.

To fully characterise debonding and sliding two variables are needed. The first one is the inelastic strain $\bar{\epsilon}_{\text {in }}$, and the second one, denoted by $d$, is introduced to define the stored energy density level $\vec{\psi}_{\mathrm{s}}$ associated with debonding and sliding alone

$\bar{\psi}_{\mathrm{s}}=\frac{E}{2} \frac{\overline{\mathrm{in}}_{\mathrm{in}}}{d}$.

The expression of $\bar{\psi}_{\mathrm{s}}$ can be found in Refs. $[16,17]$ to model concrete and rocks for which there is one damage variable modelling the stiffness reduction when the crack is open, and sliding in mode II when the same crack is closed. However, in the study of CMCs there is a mode I matrix crack as well as a mode II debond crack (Fig. 1), therefore two damage variables are needed. Furthermore, there is a non-uniform stress field along the debond crack, thus Eq. (8) does not necessarily allow the damage variable $d$ to be related to a debond crack density.

In addition to the total strain $\bar{\epsilon}$, the total free energy density $\bar{\psi}$ is dependent on three internal variables: one damage variable $D$ modelling matrix-cracking and related to the crack spacing, two variables $\bar{\epsilon}_{\text {in }}$ and $d$ describing debonding and sliding, and related to the debond length

$\bar{\psi}=\frac{E(1-D)}{2}\left(\bar{\epsilon}-\bar{\epsilon}_{\mathrm{in}}\right)^{2}+\frac{E}{2} \frac{\bar{\epsilon}_{\mathrm{in}}^{2}}{d}$.

The associated forces are defined as $\bar{\sigma}=\frac{\partial \bar{\psi}}{\partial \bar{\epsilon}}=E(1-D)\left(\bar{\epsilon}-\bar{\epsilon}_{\text {in }}\right)$,

$Y=-\frac{\partial \bar{\psi}}{\partial D}=\frac{E}{2}\left(\bar{\epsilon}-\bar{\epsilon}_{\mathrm{in}}\right)^{2}$,

$\bar{X}=-\frac{\partial \bar{\psi}}{\partial \bar{\epsilon}_{\text {in }}}=\bar{\sigma}-E \frac{\bar{\epsilon}_{\mathrm{in}}}{d}$,

$y=-\frac{\partial \bar{\psi}}{\partial d}=\frac{E}{2}\left(\frac{\overline{\overline{\mathrm{in}}}_{\mathrm{in}}}{d}\right)^{2}$.

Eq. (10) defines the macroscopic stress $\bar{\sigma}$. The following equations define the associated forces to each dissipative mechanism studied previously. Eq. (11) defines the energy release rate density $Y$ playing a similar role as the energy release rate $\mathscr{G}$ in linear elastic fracture mechanics. By combining Eqs. (10) and (11), one shows that the energy release rate density $Y$ is proportional to the square of the 'effective stress' $\bar{\sigma} /(1-D)$ [15]. Eq. (12) defines the back stress $\bar{X}$ related to debonding and sliding. Its exact value depends upon the interfacial behaviour. Eq. (13) defines the stored energy density $y$ released during debonding and sliding. Since the variable $d$ depends upon the details of the interfacial behaviour, the definition of its associated force is also dependent upon the interfacial behaviour. The damage variable $d$ and its associated force $y$ are not important in terms of overall composite behaviour. However in terms of energy contributions these variables are relevant.

Under cyclic load histories, there is an evolution of the inelastic strains $\bar{\epsilon}_{\text {in }}(N)$ and the stored energy $\bar{\psi}_{\mathrm{s}}(N)$. By inspection of Eqs. (4), (5) and (8), there is a direct influence of wear on the inelastic strain as well as on the damage variable $d(N)$. Thus to write the evolution laws during fatigue, one needs to model the evolution of wear as a function of the number of cycles $N$. When a constant stress amplitude is applied, the evolution of the interfacial shear strength $\tau$ is written as a function $N: \tau(N)=\tau_{0} \Phi(N)$ [18], where $\Phi$ is a decreasing function with the number of cycles $(\Phi(0)=1)$. The evolution of the variables $\bar{\epsilon}_{\text {in }}(N)$ and $d(N)$ can be related to their monotonic values $\bar{\epsilon}_{\text {in }}$ and $d$ reached during the first loading $(N=0)$ by

$\bar{\epsilon}_{\text {in }}(N)=\frac{\bar{\epsilon}_{\text {in }}}{\Phi(N)}, \quad d(N)=\frac{d}{\Phi(N)}$

and the corresponding stored energy density $\bar{\psi}_{\mathrm{s}}(N)$ can be written as

$\bar{\psi}_{\mathrm{s}}(N)=\frac{\bar{\psi}_{\mathrm{s}}}{\Phi(N)}$

where $\bar{\psi}_{\mathrm{s}}$ is the initial value of the stored energy. Eq. (15) shows that the stored energy density increases as do the inelastic strains and the damage variable (see Eq. (14)). 


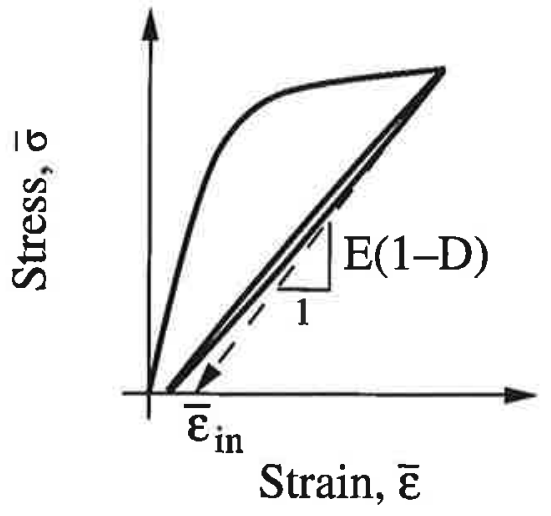

Fig. 2. Schematic stress-strain curve.

To determine the evolution laws of the internal variables, two methods can be followed. The first one is using simulations of the complete micromechanical model along the lines developed for instance by Feillard et al. [19] to get the evolutions of the crack spacing $2 L$ and the debond length $2 l_{\mathrm{d}}$, and then the state potential. The second one is by performing experiments. The damage variable $D$ is obtained by measuring the initial unloading modulus and the corresponding inelastic strain is $\bar{\epsilon}_{\text {in }}$ (Fig. 2). To measure the variable $d$, the stored energy $\bar{\psi}_{\mathrm{s}}$ has to be evaluated, for instance by using methods developed by Chrysochoos [20] or Cho et al. [21].

\section{Generalisation to $[0,90]$ composites}

A 3D formulation of matrix-cracking requires an anisotropic damage description [12]. In the case of cracking perpendicular to the fibre direction in a unidirectional composite, the generalisation is straightforward since the only compliance change is given in the fibre direction, and therefore only one scalar anisotropic damage variable is needed and the previous 1D analysis is still relevant. For a $[0,90]$ layered composite, the reversible part of the Helmholtz free energy density, which is the Legendre transformation of the corresponding Gibbs' specific enthalpy, depends upon the damage variables modelling matrix-cracking in the 0 and $90^{\circ}$ layers

$\bar{\psi}_{\mathrm{c}}=\frac{1}{2} \overline{\underline{\epsilon}}_{\mathrm{e}}: \underline{\underline{\underline{\underline{\underline{E}}}}}\left(D_{\mathrm{m}}^{0}, D_{\mathrm{m}}^{90}\right): \overline{\underline{\underline{\epsilon}}}_{\mathrm{e}}$

where $\overline{\underline{\epsilon}}_{e}$ denotes the elastic strain tensor, ' $\because$ ' the contraction with respect to two indices, $\underline{E}$ the elastic stiffness tensor which is a function of the matrix-cracking damage variables in the $0^{\circ}$ ply (i.e. $D_{\mathrm{m}}^{0}$ ) and the $90^{\circ}$ ply (i.e. $D_{\mathrm{m}}^{90}$ ) [12].

In a 3D formulation of interfacial debonding and sliding, a second-order tensor is needed to model the inelastic strains. In the case of a unidirectional composite, when cracking occurs perpendicularly to the fibre direction, only one inelastic strain component is different from zero, viz. the normal component along the fibre direction. Similarly, only one scalar debond damage variable is needed. For a layered $[0,90]$ composite, a first-order approximation requires only the in-plane components $\left(\bar{\epsilon}_{\mathrm{in} 11}, \bar{\epsilon}_{\mathrm{in} 22}\right.$ and $\left.\bar{\epsilon}_{\mathrm{in} 12}\right)$ of the inelastic strain tensor $\bar{\epsilon}_{i n}$. Since each operative slip system can be integrated separately in terms of energetic contributions, the internal damage variables can be defined separately for each inelastic strain term. Therefore there are as many debond damage terms as non-vanishing inelastic strain components [12] to define the stored energy density

$\bar{\psi}_{\mathrm{s}}=\frac{E_{11}}{2} \frac{\overline{\mathrm{e}}_{\mathrm{in} 11}^{2}}{d_{11}}+\frac{E_{22}}{2} \frac{\bar{\epsilon}_{\mathrm{in} 22}^{2}}{d_{22}}+\frac{G_{12}}{2} \frac{\bar{\epsilon}_{\mathrm{in} 12}^{2}}{d_{12}}$

where $E_{11}, E_{22}$, are the Young's moduli along the 1-direction and the 2-direction, respectively, and $G_{12}$ is the shear modulus in the 1-2 plane. The state potential $\bar{\psi}$ is the sum of the two previous energy densities

$$
\begin{aligned}
& \bar{\psi}=\frac{1}{2}\left(\underline{\underline{\bar{\epsilon}}}-\overline{\underline{\epsilon}}_{i n}\right): \underline{\underline{\underline{\underline{E}}}}\left(D_{\mathrm{m}}^{0}, D_{\mathrm{m}}^{90}\right):\left(\underline{\underline{\underline{\bar{\epsilon}}}}-\underline{\underline{\underline{\epsilon}}}_{i n}\right)+\frac{E_{11}}{2} \frac{\bar{\epsilon}_{i n 11}^{2}}{d_{11}}+\frac{E_{22}}{2} \frac{\bar{\epsilon}_{\text {in22 }}^{2}}{d_{22}} \\
& +\frac{G_{12}}{2} \frac{\bar{\epsilon}_{\mathrm{in} 12}^{2}}{d_{12}}
\end{aligned}
$$

and the associated forces are defined as follows

$\underline{\underline{\bar{\sigma}}}=\frac{\partial \bar{\psi}}{\partial \underline{\underline{\underline{\epsilon}}}}$,

$Y_{\mathrm{m}}^{0}=-\frac{\partial \bar{\psi}}{\partial D_{\mathrm{m}}^{0}}, \quad Y_{\mathrm{m}}^{90}=-\frac{\partial \bar{\psi}}{\partial D_{\mathrm{m}}^{90}}$,

$\underline{\underline{\underline{X}}}=-\frac{\partial \bar{\psi}}{\partial \overline{\underline{\epsilon}}_{\text {in }}}$,

$y_{11}=-\frac{\partial \bar{\psi}}{\partial d_{11}}, \quad y_{22}=-\frac{\partial \bar{\psi}}{\partial d_{22}}, \quad y_{12}=-\frac{\partial \bar{\psi}}{\partial d_{12}}$.

Eqs. (19)-(22) are the generalisation of Eqs. (10)-(13) in the case of a $[0,90]$ composite.

The remaining part of the model is concerned with the evolution laws. The evolution law for matrix-cracking damage $\left(D_{\mathrm{m}}=D_{\mathrm{m}}^{0}\right.$ or $\left.D_{\mathrm{m}}^{90}\right)$ is expressed as a function of the associated force $\left(Y_{\mathrm{m}}=Y_{\mathrm{m}}^{0}\right.$ or $\left.Y_{\mathrm{n}}^{90}\right)$. It is obtained by analysing tensile tests at $45^{\circ}$ on a $[0,90]$ composite (for which $D_{\mathrm{m}}^{0}=D_{\mathrm{m}}^{90}$ ). A suitable expression is given by

$D_{\mathrm{m}}=D_{\mathrm{sat}}\left(1-\exp \left[-\left(\frac{Y_{\mathrm{m}}}{\mathrm{Y}_{\mathrm{m} 0}}\right)^{m_{\mathrm{m}}}\right]\right)$

where $D_{\text {sat }}$ is the saturation value of the damage parameter modelling matrix-cracking, $Y_{\mathrm{m} 0}$ a scale parameter and $m_{\mathrm{m}}$ a shape parameter.

The evolution law of the inelastic strain is written as 
$\bar{\epsilon}_{\text {in11 }}=\frac{\left\langle\bar{\sigma}_{11}-\sigma_{\mathrm{th}}\right\rangle}{\sigma_{0}}, \quad \bar{\epsilon}_{\mathrm{in} 22}=\frac{\left\langle\bar{\sigma}_{22}-\sigma_{\mathrm{th}}\right\rangle}{\sigma_{0}}$

where \langle\rangle denotes the positive part of the considered quantity, $\sigma_{\mathrm{th}}$ a threshold stress below which no inelastic strains are observed and $\sigma_{0}$ a scale parameter.

The evolution law of the inelastic strain $\bar{\epsilon}_{\text {in12 }}$ is expressed as

$\bar{\epsilon}_{\text {in } 12}=\frac{\left\langle\bar{\tau}_{\mathrm{cq}}-\tau_{\mathrm{th}}\right\rangle}{\tau_{0}} \operatorname{Sign}\left(\bar{\sigma}_{12}\right)$

where $\tau_{\mathrm{th}}$ is a threshold stress and $\tau_{0}$ a scale parameter. The introduction of the equivalent shear stress $\bar{\tau}_{\text {eq }}$ comes from the fact that the stress-strain curve in tension at $45^{\circ}$ is usually very close to that in shear for most CMCs [22]. The following expression complies with the previous requirement

$\bar{\tau}_{\mathrm{eq}}=\sqrt{\left\langle\bar{\sigma}_{12}\right\rangle\left[\left\langle\bar{\sigma}_{12}\right\rangle+\frac{3}{2}\left\langle\bar{\sigma}_{11}+\bar{\sigma}_{22}\right\rangle\right]}$.

This equivalent stress can also be used in the study of geomaterials for which the inelastic strains are driven by the shear stress as well as the hydrostatic stress [23].

When the cyclic stress amplitude does not vary significantly, the evolution of the inelastic strain is modelled by a function $\Phi$ introduced in the 1D analysis. The result is generalised so that the evolution of the inelastic strain is given by

$$
\begin{aligned}
& \bar{\epsilon}_{\text {in } 11}(N)=\frac{\bar{\epsilon}_{\text {in } 11}}{\Phi_{1}(N)}, \quad \bar{\epsilon}_{\text {in } 22}(N)=\frac{\bar{\epsilon}_{\text {in } 22}}{\Phi_{1}(N)}, \\
& \bar{\epsilon}_{\text {in } 12}(N)=\frac{\bar{\epsilon}_{\text {in } 12}}{\Phi_{2}(N)} .
\end{aligned}
$$

The functions $\Phi_{i}$ are modelled by

$\Phi_{i}(N)=1-\gamma_{i}\left[1-\exp \left\{\left[\ln \left(\frac{N+1}{N_{0 i}}\right)\right]^{\alpha_{i}}\right\}\right]$ with $i=1,2$

where $\alpha_{i}, \gamma_{i}$ and $N_{0 i}$ are material parameters. The identification is performed by using one experiment at $0^{\circ}$ (to determine $\Phi_{1}$ ) and another one at $45^{\circ}$ (to get $\Phi_{2}$ ). In the following section the model will be applied to a $[0,90]$ $\mathrm{SiC} / \mathrm{SiC}$ composite.

\section{Application to a $[0,90] \mathrm{SiC} / \mathrm{SiC}$ composite}

The identification is performed on a $[0,90]_{\mathrm{S}}$ layered $\mathrm{SiC} / \mathrm{SiC}$ composite by using the experimental data of Pluvinage [24]. To identify the material parameters, two tensile tests at 0 and $45^{\circ}$ are used. The material parameters modelling matrix-cracking are obtained by analysing sequences of loading-partial unloading (see Fig. 2). The measure of the initial unloading modulus of a tensile test at $45^{\circ}$ allows the evolution law of the damage variables $\left(D_{\mathrm{m}}^{0}=D_{\mathrm{m}}^{90}\right)$ to be determined. The following parameters are obtained

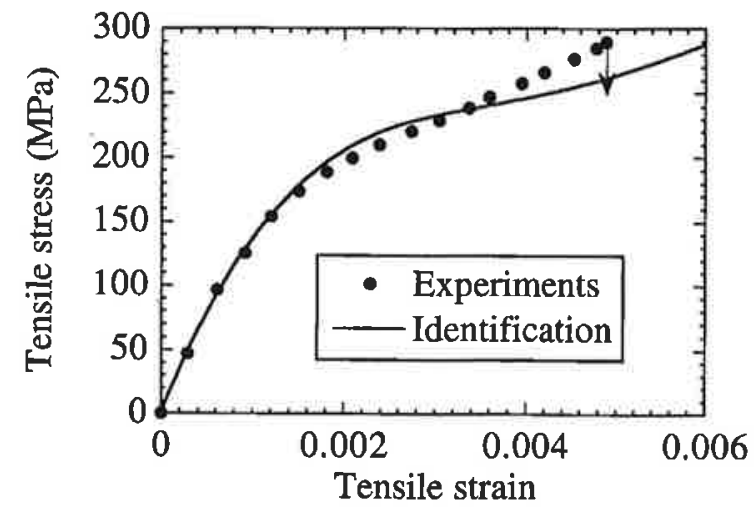

Fig. 3. Stress-strain curve at $0^{\circ}$ for a $\mathrm{SiC} / \mathrm{SiC}$ composite.

$D_{\text {sat }}=1.0, \quad Y_{\mathrm{m} 0}=0.60 \mathrm{MPa}, \quad m_{\mathrm{m}}=1.2$.

The evolution law of the inelastic strains in the fibre directions are determined by considering the tensile test at $0^{\circ}$. The knowledge of the damage evolution laws enables us to identify the following parameters

$\sigma_{\mathrm{th}}=88 \mathrm{MPa}, \quad \sigma_{0}=4.8 \mathrm{GPa}$.

The analysis of the tensile test at $45^{\circ}$ allows us to identify the last parameters needed to model the inelastic strain $\bar{\epsilon}_{\text {in12 }}$

$\tau_{\mathrm{th}}=77 \mathrm{MPa}, \quad \tau_{0}=324 \mathrm{MPa}$.

The experimental and identified stress-strain response is plotted in Figs. 3 and 4. There is a good agreement between experimental and identified stress-strain curves. A validation on a Iosipescu experiment can be found in Ref. [22].

To carry out structural calculations, the model presented here has been implemented in the industrial code ABAQUS [25] via a user material UMAT subroutine. The previous data are used to study a plate containing a hole and loaded in remote tension. The composite is oriented at $0^{\circ}$ with respect to the loading direction. Fig. 5 shows the mesh used in the finite element analysis. In Fig. 6 the stress profile along the ligament is plotted as

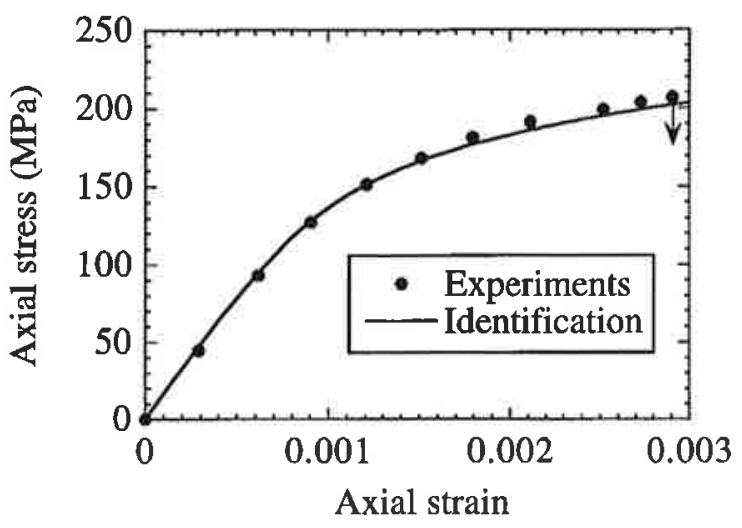

Fig. 4. Stress-strain curve at $45^{\circ}$ for a $\mathrm{SiC} / \mathrm{SiC}$ composite. 


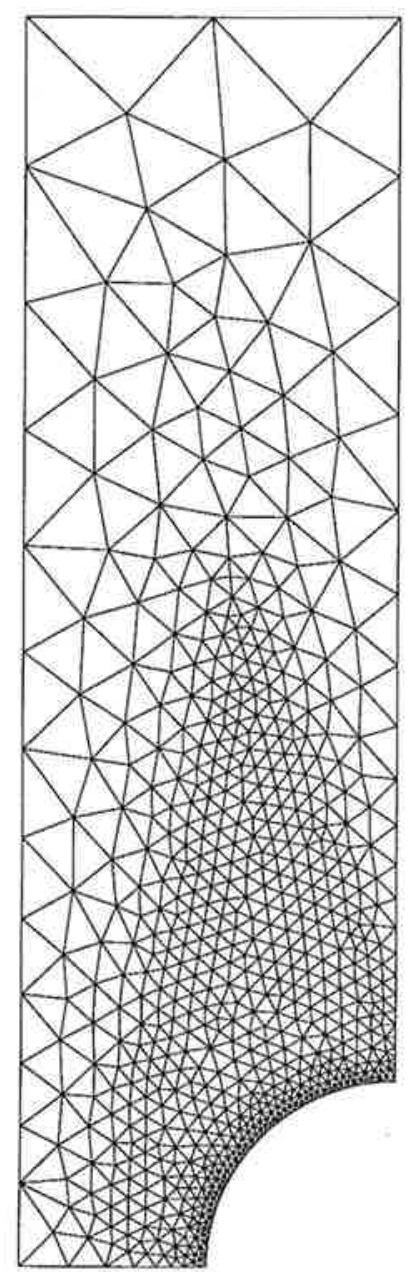

Fig. 5. Mesh of a plate with a hole made of a $[0,90] \mathrm{SiC} / \mathrm{SiC}$ composite loaded in remote tension at $0^{\circ}$.

a function of the applied load level. The stress redistribution under monotonic loading is compared with an elastic computation. One can observe that the stress redistribution increases as the load level increases lead-

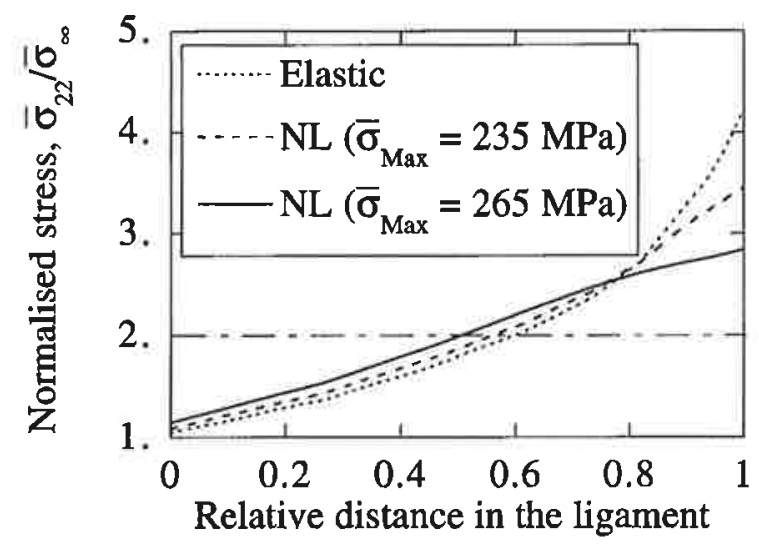

Fig. 6. Tensile stress profile in the ligament of a plate with a hole loaded in remote tension for an elastic computation, a non-linear computation $\left(\bar{\sigma}_{\mathrm{Max}}=325 \mathrm{MPa}\right.$ and $\left.\bar{\sigma}_{\mathrm{Max}}=265 \mathrm{MPa}\right)$. ing to a reduction of the stress concentration factor in the vicinity of the hole. Fig. 7 shows the contours of the damage variable $D_{\mathrm{m}}^{0}$, the normal stress along the loading direction $\bar{\sigma}_{22}$, the in-plane shear stress $\bar{\sigma}_{12}$, and the normal inelastic strain along the loading direction $\bar{\epsilon}_{\text {in22 }}$. The shear stresses are relatively small in comparison with the normal stresses so that it is expected that the failure mode will be tensile (mode I). The presence of damage and inelastic strains induces the stress redistribution shown in Fig. 6 .

To identify interfacial wear, cyclic experiments are needed. The only experiments available on the same material were carried out in four-point bending (inner span: $28 \mathrm{~mm}$, outer span: $84 \mathrm{~mm}$, beam height: $2.3 \mathrm{~mm}$, beam width: $10 \mathrm{~mm}$ ). The measurements and the identification are plotted in Fig. 8 and correspond to following parameters

$\alpha_{1}=1.6, \quad \gamma_{1}=0.98, \quad N_{01}=7$.

The maximum value of the inelastic strain is used for the identification of the function $\Phi_{1}(N)$. The minimum value of the inelastic strain is a prediction by assuming that the interfacial behaviour is modelled by a constant shear strength. In that case, the minimum value is half of the maximum value [26]. Fig. 8 shows that the predictions agree well with the experimental measurements of the minimum value of the inelastic strain.

In Fig. 9 the stress profile in the mid-section is plotted as a function of the number of cycles. Again there is a stress redistribution due to non-linearity under monotonic loading condition $(N=0)$. However the stress redistribution is small enough to allow the assumption of a constant stress amplitude for $N>0$. In the present case most of the stress redistribution is due to the first cycle. This is due to the small level of inelastic strains in the $\mathrm{SiC} / \mathrm{SiC}$ composite studied in this paper. Furthermore, the whole beam height is affected by the stress distribution. This result distinguishes the beam from the plate with a hole.

Lastly, to analyse a plate with a hole, the function $\Phi_{2}(N)$ is needed. A cyclic tensile at $45^{\circ}$ test is needed to identify the missing function. These data are not yet available for the $\mathrm{SiC} / \mathrm{SiC}$ composite analysed in this paper.

\section{Conclusions}

A continuum damage mechanics formulation has been applied to unidirectional ceramic matrix composites. The internal variables which define the state of the material, in addition to the total strain, have been identified. Matrix-cracking is described by one damage variable, debonding and sliding are modelled by an inelastic strain and by another damage variable measuring the amount of stored energy. These variables are 

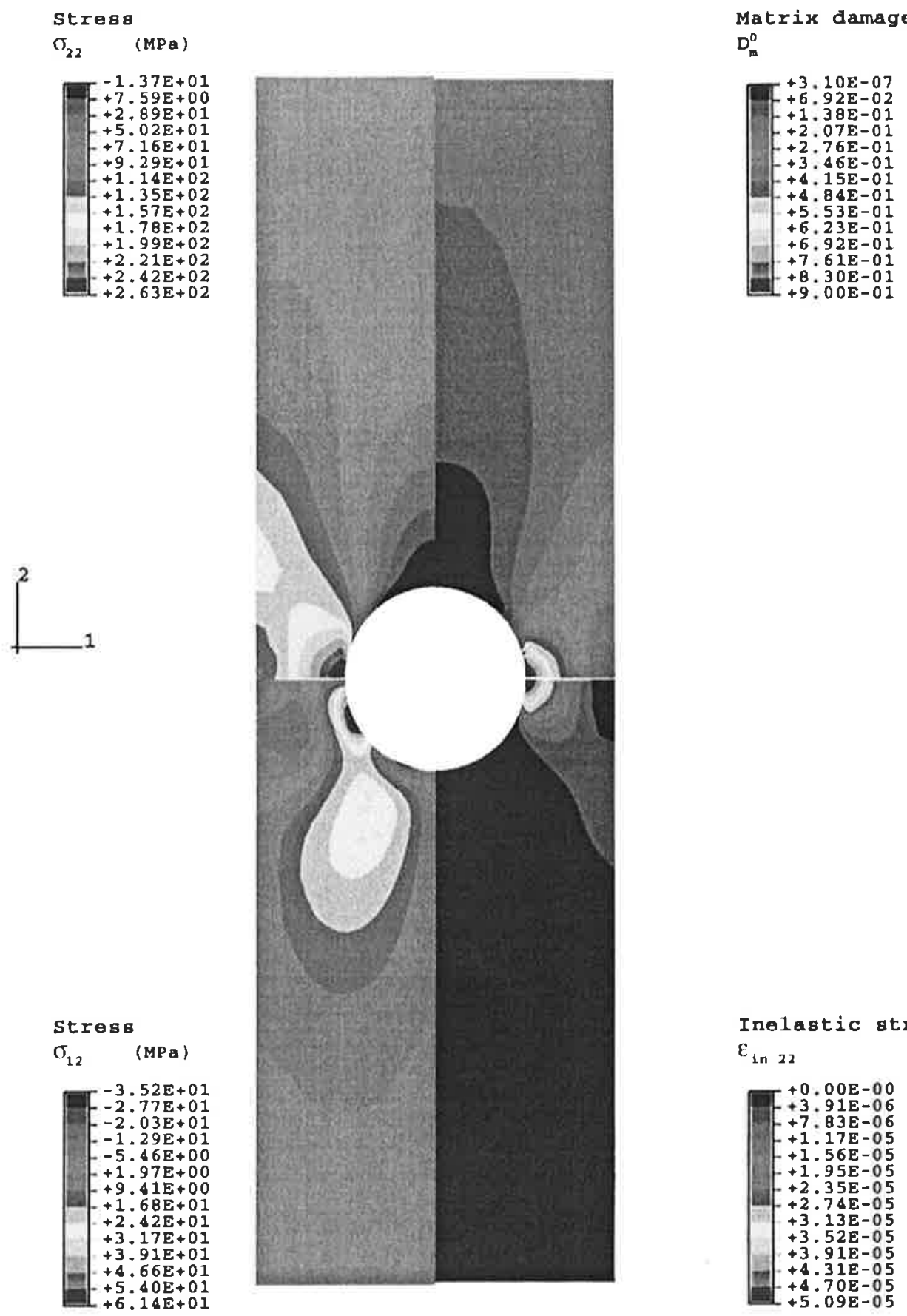

Inelastic strain

$\varepsilon_{\ln 22}$

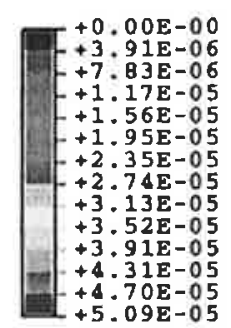

Fig. 7. Contours of damage variable modelling matrix-cracking $\left(D_{\mathrm{n}}^{0}\right)$, normal stress $\bar{\sigma}_{22}$, shear stress $\bar{\sigma}_{\mathrm{I} 2}$ and normal inelastic strain $\bar{\epsilon}_{\text {in22 }}$ in a plate with a hole loaded in remote tension $\left(\bar{\sigma}_{\mathrm{Max}}=265 \mathrm{MPa}\right)$.

related to microscopic quantities introduced to analyse the degradation mechanisms of ceramic matrix composites. It is also shown that interfacial wear can be modelled by using the above-mentioned variables.

The previous framework is then extended to model layered as well as woven ceramic matrix composites. The nature of the different internal variables is discussed. A state potential and the evolution laws are introduced. It is shown that when fibre breakage is not modelled, five internal variables are needed to describe the stress-strain behaviour of $[0,90]$ composites. Under monotonic loading conditions, three different evolution laws are identified. Under cyclic loading conditions, two additional functions need to be determined.

The parameter identification is carried out on a $\mathrm{SiC}_{/}$ $\mathrm{SiC}[0,90]$ composite. Only two tensile tests are needed, viz. one at $0^{\circ}$ and one at $45^{\circ}$. The monotonic as well as the cyclic behaviour are identified. The stress redistribution is analysed in bending as well as on a plate containing a hole and loaded in remote tension. It is 


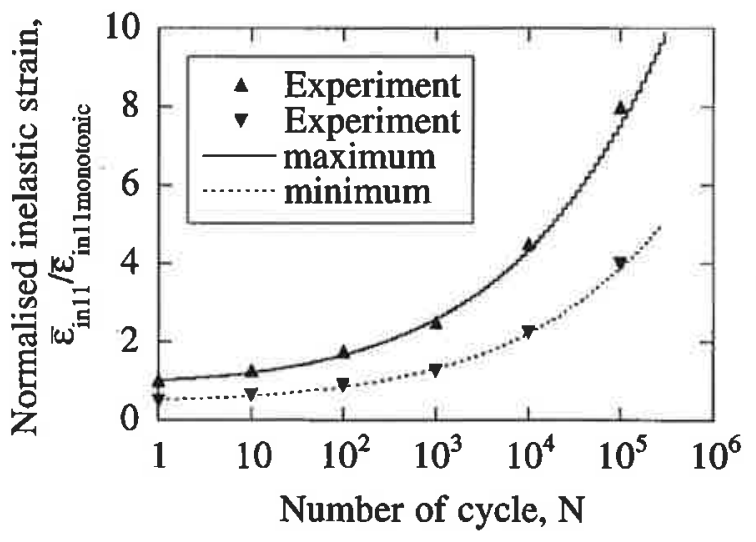

Fig. 8. Maximum and minimum inelastic strain $\bar{\epsilon}_{\text {in } 11}$ normalised by the inelastic strain under monotonic loading $\bar{\epsilon}_{\text {in IImonotonic }}$ as a function of the number of cycles $N$ in four-point bending for a $\mathrm{SiC} / \mathrm{SiC}$ composite. Comparison between experimental and identified data. The strains are measured at the tensile surface of the mid-point.

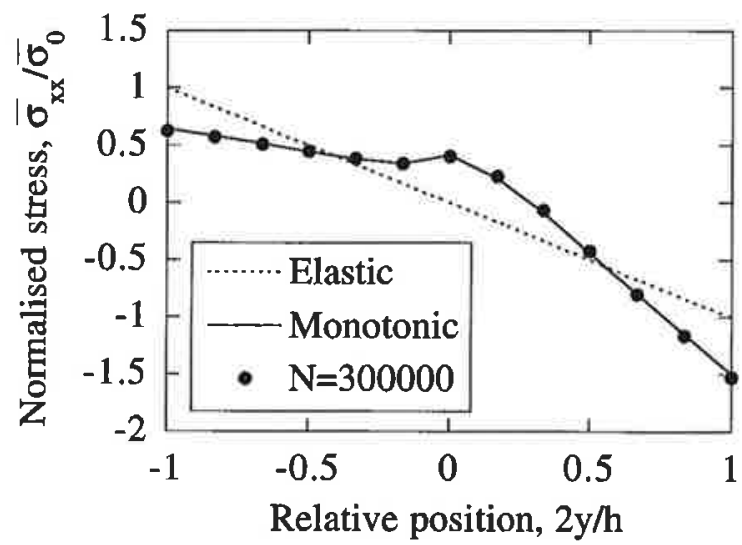

Fig. 9. Tensile stress profile at the mid-section of the beam loaded in four-point bending when $N=0$ (elastic computation), $N=0$ (non-linear computation) and $N=3 \times 10^{5}$ cycles (non-linear computation).

shown that the redistribution induced by 'cyclic rachetting' is less significant than that caused by the non-linear monotonic behaviour in the case of beam bending.

\section{Acknowledgements}

This work was supported by the Advanced Research
Projects Agency through the University Research Initiative, ONR Contract N00014-12-J-1808.

\section{References}

[1] D. Beyerle, S.M. Spearing, F.W. Zok, A.G. Evans, J, Am. Ceram. Soc. 75 (10) (1992) 2719-2725.

[2] A.W. Pryce, P.A. Smith, J. Mater. Sci. 27 (1992) 2695-2704.

[3] D. Rouby, P. Reynaud, Comp. Sci. Tech. 48 (1993) 109-118.

[4] I. Prigogine, Introduction to Thermodynamics of Irreversible Processes, Wiley, New York, 1962.

[5] D.B. Coleman, M.E. Gurtin, J. Chem. Phys. 47 (1967) 597-613.

[6] V. Volterra, Ann. Sci. l'Ec. Norm. Supér., Paris 24 (3) (1907) $401-518$.

[7] F. Hild, A. Burr, F.A. Leckie, Eur, J. Mech. A/Solids 13 (6) (1994) $731-749$

[8] F. Hild, P.-L. Larsson, F.A. Leckie, Comp. Struct. 29 (1994) $365-377$.

[9] J. Aveston, A. Kelly, J. Mater. Sci. 8 (1973) 352-362.

[10] J. Aveston, G.A. Cooper, A. Kelly, Proceedings National Physical Laboratory: Properties of Fiber Composites, IPC Science and Technology Press, Surrey, UK, 1971, pp. 15-26.

[11] J.W. Hutchinson, H.M. Jensen, Mech. Mater. 9 (1990) 139-163

[12] A. Burr, F. Hild, F.A. Leckie, Arch. Appl. Mech. 65 (7) (1995) 437-456.

[13] J. Lemaitre, J. Dufailly, Proc. $3^{\text {e }}$ congrès français de mécanique 1977.

[14] J. Lemaitre, J. Dufailly, Eng. Fract. Mech. 28 (5-6) (1987) $643-661$.

[15] J Lemaitre, A Course on Damage Mechanics, Springer-Verlag, Berlin, 1992

[16] S. Andrieux, C.R. Acad. Sci. Paris, Série II t. 293 (1981) $329-332$.

[17] S. Andrieux, Y. Bamberger, J.-J. Marigo, J. Méc. Th. Appl. 5 (3) (1986) 471-513

[18] A.G. Evans, F.W. Zok, R.M. McMeeking, Acta Metall. Mater. 43 (3) (1995) $859-875$.

[19] P. Feillard, G. Désarmot, J.-P. Favre, Comp. Sci. Tech. 50 (1994) 265-279.

[20] A. Chrysochoos, O. Maisonneuve, G. Martin, H. Caumon Nucl. Eng. Des. 114 (1989) 323-333.

[21] C. Cho, J.W. Holmes, J.R. Barber, J. Am. Ceram. Soc. 74 (11) (1991) 2802-2808.

[22] A. Burr, F. Hild, F.A. Leckie, Eur, J. Mech. A/Solids 16 (1) (1997) 53-78.

[23] D.C. Drucker, W. Prager, Quat. Appl. Math., 14 (1956).

[24] P. Pluvinage, Ph.D. Dissertation, University Bordeaux I, 1991,

[25] H.D. Hibbitt, B.I. Karlsson, P. Sorensen, Abaqus version 5.5, 1995.

[26] F. Hild, A. Burr, F.A. Leckie, Int. J. Solids Struct. 33 (8) (1996) $1209-1220$. 